\title{
New insights into the stem cell organization of Barrett esophagus
}

New research has demonstrated that Barrett metaplastic glands are clonal and contain multiple stem cells. These findings are important as although Barrett esophagus is known to develop as a result of chronic gastroesophageal reflux, not much is known about the cellular origin of this disease and how it spreads.

Stuart McDonald, Janusz Jankowski and colleagues decided to use mutations in the mitochondrial genome (mtDNA) as a genetic marker to investigate stem cell organization in Barrett metaplasia. "Only stem cells survive for a sufficient period of time to allow a single mutation in a single copy of mtDNA to divide and become fixed within the cell," explain the authors. These mtDNA mutations are also useful markers because they do not confer any proliferative or antiapoptotic advantage over neighboring wild-type cells.

Deficiency of cytochrome $c$ oxidase (CCO; a mitochondrially encoded enzyme) is often associated with mtDNA mutations and is therefore a useful indicator of whether these mutations are present. The researchers have previously applied these techniques to investigate stem cell populations in intestinal crypts and gastric glands. Patches of CCO-deficient cells that shared the same mtDNA mutation revealed clonal expansion of these stem cell populations.

The researchers have now turned their attention to Barrett esophagus and used the same methods to analyze tissue specimens from 11 patients with this disease. The main findings are that Barrett metaplastic glands are clonal-each area

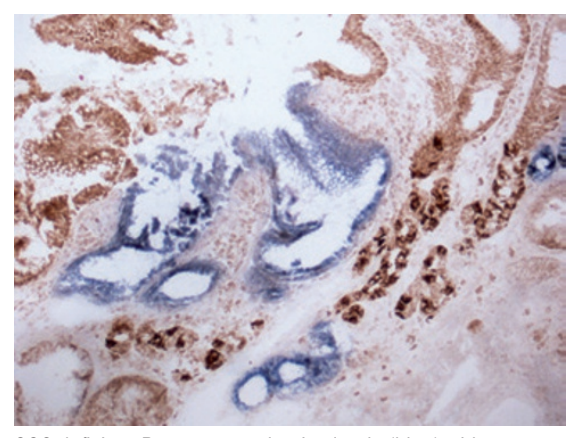

cco-deficient Barrett metaplastic glands (blue) with CCO-normal neighbors (brown). Courtesy of S. McDonald. within a CCO-deficient patch contained cells with identical mtDNA mutations. However, adjacent glands were not necessarily derived from the same clone, indicating the presence of multiple stem cells. The researchers were also able to show that squamous and columnar cells originate from the same population of stem cells, that is, a common stem cell is capable of producing both cell types.

"We see no reason why this approach cannot be used to investigate the location and architecture of the stem cell niche in other tissues," say McDonald and Jankowski. "With regards to Barrett esophagus, work is underway to measure CCO-deficient gland patch size compared with patient age to determine the rate of expansion of Barrett metaplastic glands."

\section{Isobel Franks}

Original article Nicholson, A. M. et al. Barrett's metaplasia glands are clonal, contain multiple stem cells and share a common squamous progenitor. Gut doi:10.1136/ gutjnl-2011-301174 